\title{
SEROPREVALENCE AND RISK FACTORS OF BESNOITIA BESNOITI INFECTION IN KOREAN CATTLE - SHORT COMMUNICATION
}

\author{
Seung-Hun LEE ${ }^{1,2}$, Kyung-Yeon EO ${ }^{3}$, Byeong Yeal JuNG ${ }^{4}$, Dongmi KwAK ${ }^{1}$ \\ and Oh-Deog KwON ${ }^{1 *}$ \\ ${ }^{1}$ College of Veterinary Medicine, Kyungpook National University, Daegu 41566, Korea; \\ ${ }^{2}$ Foreign Animal Disease Division, Animal and Plant Quarantine Agency, \\ Gimcheon, Korea; ${ }^{3}$ Conservation and Health Center, Seoul Zoo, Gwacheon, Korea; \\ ${ }^{4}$ Bacterial Disease Division, Animal and Plant Quarantine Agency, Gimcheon, Korea
}

(Received 7 March 2017; accepted 6 November 2017)

\begin{abstract}
Besnoitia besnoiti is an obligate intracellular parasite that is transmitted by direct contact or via mechanical transmission by flies as vectors. Besnoitiosis causes economic losses in the cattle industry and is regarded as a re-emerging disease in Europe. This study evaluated the seroprevalence of $B$. besnoiti in Korean cattle using a commercial ELISA kit. Among 558 serum samples, 19 (3.4\%) tested seropositive for $B$. besnoiti. The statistically significant risk factors included age ( $\geq 2$ years), sex (castrated males), and region (lower latitudes) $(\mathrm{P}<0.05)$. The overall seroprevalence suggested a wide distribution of $B$. besnoiti infection in cattle reared in Korea. Thus, the practice of intensive cattle husbandry and the regionally different seroprevalence of $B$. besnoiti infection in cattle in Korea warrant routine monitoring and vector control to reduce economical losses due to bovine besnoitiosis in the country.
\end{abstract}

Key words: Besnoitia besnoiti, cattle, re-emerging disease, seroprevalence, vector-borne disease

Besnoitia besnoiti (Apicomplexa: Sarcocystidae) is an obligate intracellular parasite that causes bovine besnoitiosis (Cortes et al., 2014). In contrast to other coccidian parasites, the main route of spreading of $B$. besnoiti between animals is mechanical transmission by flies as vectors (Hornok et al., 2015). The clinical signs of besnoitiosis in cattle differ according to the stage of infection. Pyrexia, nasal and ocular discharge, salivation, stiff gait, and subcutaneous oedema are observed in the acute stage (Schares et al., 2013). In the chronic stage, severely lichenified and alopecic skin is observed, and the bulls can develop orchitis, resulting in infertility (Schares et al., 2013). Generally, while bovine

*Corresponding author; E-mail: odkwon@knu.ac.kr; Phone: 0082 (53) 950-5960; Fax: 0082 (53) 950-5955 
besnoitiosis is associated with a high morbidity and low mortality, not all infected animals develop clinical signs (Jacquiet et al., 2010; Hornok et al., 2015).

Bovine besnoitiosis has been reported worldwide, including countries in Africa, Asia, America, and Europe (Lee et al., 1970; EFSA, 2010; Olias et al., 2011; Rinaldi et al., 2013; Cortes et al., 2014; Álvarez-García et al., 2014; Ashmawy and Abu-Akkada, 2014; Talafha et al., 2015). Many studies were published before 1992, and the disease apparently receded between 1992 and 2001 (Olias et al., 2011). However, since 2002, besnoitiosis has been reported frequently in Europe and has been recognised as a re-emerging and endemic disease by the European Food and Safety Authority (EFSA, 2010).

Cattle are among the most economically significant livestock in Korea, where the estimated cattle population approaches three million (Oh et al., 2016). Only few studies have examined the prevalence of besnoitiosis in Korea, and most of them were published several decades ago (Lee et al., 1970; Lee, 1975). The purpose of this study was to evaluate the current serologic distribution of $B$. besnoiti in cattle reared in Korea and to analyse its epidemiologic aspects.

\section{Materials and methods}

\section{Sample collection and study area}

Whole jugular venous blood samples $(\mathrm{n}=558)$ collected from cattle throughout Korea from 2010 to 2015 were tested (Fig. 1). Serum samples were separated from the whole blood by centrifugation and stored at $-20^{\circ} \mathrm{C}$ until use. Age ( $\leq 1$ year, young; $\geq 2$ years, adults), sex (male, female, and castrated animals), type of cattle (beef and dairy), and region (northern, central, southern, and Jeju Island) were recorded. The geographic location of Korea ranged from $33^{\circ} 06^{\prime}$ to $38^{\circ} 27^{\prime}$ northern latitude and $125^{\circ} 04^{\prime}$ to $131^{\circ} 52^{\prime}$ eastern longitude. The mean annual temperature was $12.7^{\circ} \mathrm{C}$ (range: $8.2-17.9^{\circ} \mathrm{C}$ ) and the annual precipitation was $1500 \mathrm{~mm}$ (Lee et al., 2016). In Korea, lower latitude regions are warmer and have more precipitation (Lee et al., 2015).

\section{Sample size determination}

The sample size was determined by a simple random sampling method, with $95 \%$ confidence, $10 \%$ expected prevalence, and $5 \%$ desired absolute precision (Thrusfield, 2005). The expected prevalence was estimated based on previous reports of prevalence in Korea (Lee et al., 1970).

\section{Serological examination}

A commercial enzyme-linked immunosorbent assay (ELISA) kit (ID Screen ${ }^{\circledR}$ Besnoitia Indirect, IDvet, France) was used according to the manufacturer's in- 
structions to detect anti- $B$. besnoiti antibodies. Positive and negative controls provided by the manufacturer were included in each experiment. Doubtful results were considered negative for statistical analysis. The sensitivity and specificity of the ELISA kit have been reported as $97.2 \%$ and $100 \%$, respectively (GarcíaLunar et al., 2013).

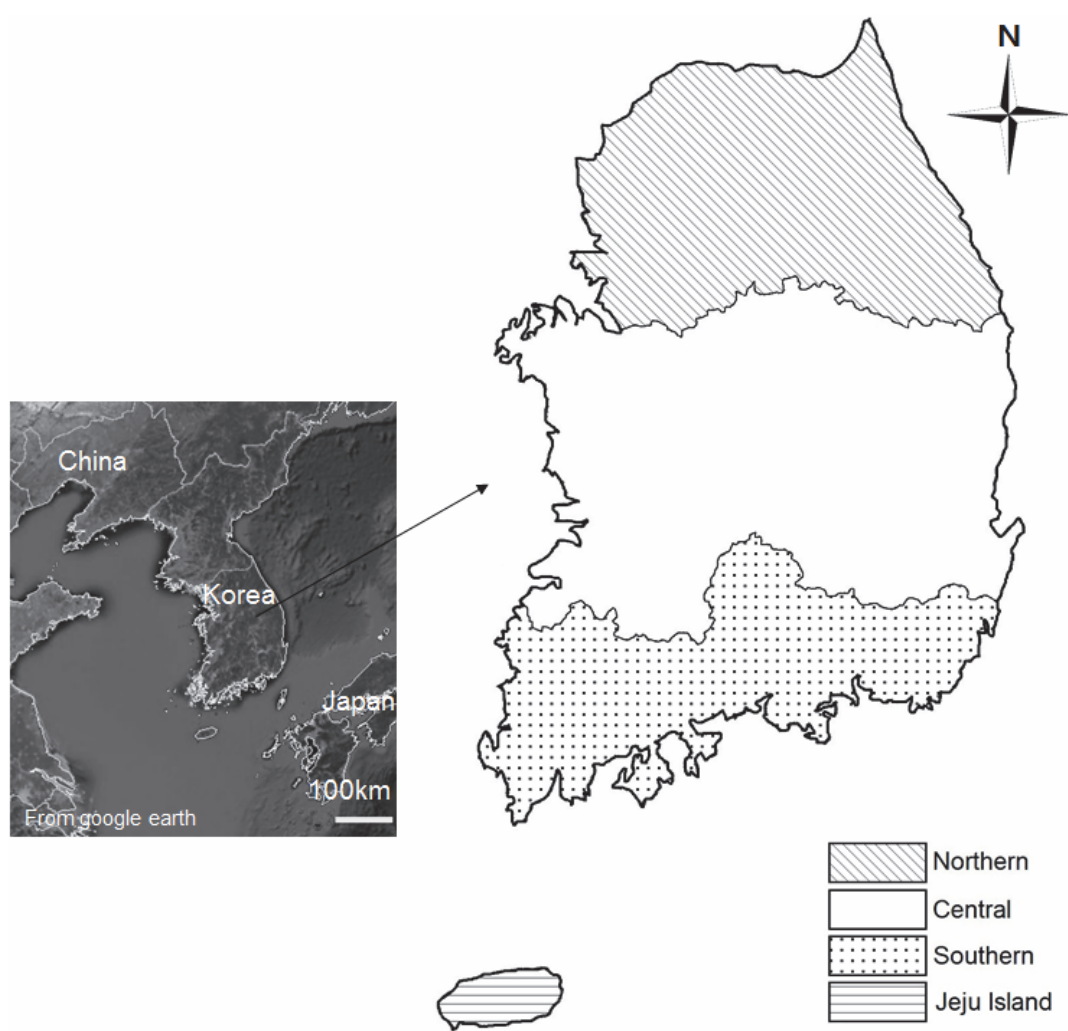

Fig. 1. The study regions in Korea. Seropositivity for anti-Besnoitia besnoiti antibodies among cattle was analysed by region. The regions are grouped according to administrative boundaries

Due to imperfection of the diagnostic method, the observed prevalence (OP; OP $=$ No. positives/No. of total samples) and true prevalence $(\mathrm{TP} ; \mathrm{TP}=$ OP + Sensitivity $-1 /$ Sensitivity + Specificity -1 ) were calculated. The $95 \%$ confidence intervals were calculated for all estimates using Blaker's method (Reiczigel et al., 2010).

\section{Statistical analysis}

Seroprevalence was analysed to evaluate association with age, sex, type of cattle, and region using the chi-squared test or Fisher's exact test. P values $<0.05$ were considered statistically significant. 


\section{Results}

Among 558 bovine serum samples, 19 (3.4\%; TP 3.5\%) and 2 (0.4\%) tested positive and doubtful, respectively, for anti- $B$. besnoiti antibodies (Table 1). Seropositivity was $1.0 \%(1 / 103$; TP $1.0 \%)$ among young cattle and $4.0 \%(18 / 455$; TP $4.1 \%)$ in adult cattle; seropositivity was $0 \%(0 / 59$; TP $0 \%)$ among male cattle, $1.9 \%$ (6/319; TP 2.0\%) among female cattle, and 7.2\% (13/180; TP 7.4\%) among castrated cattle. Beef cattle had 4.1\% (14/340; TP 4.2\%) seropositivity and dairy cattle had 2.3\% (5/218; TP 2.4\%) seropositivity. With regard to analysis based on the region, the seropositivity rates were $0.5 \%(1 / 188$; TP $0.5 \%), 4.0 \%(6 / 151$; TP 4.1\%), 7.1\% (8/112; TP 7.3\%), and 3.7\% (4/107; TP 3.8\%) for northern, central, and southern regions, and the Jeju Island, respectively. Age, sex, and region showed statistically significant $(\mathrm{P}<0.05)$ correlation with seroprevalence (Table 1$)$.

\section{Table 1}

Seroprevalence of Besnoitia besnoiti among cattle in Korea

\begin{tabular}{|c|c|c|c|c|c|c|c|c|}
\hline Variable & Group & $\begin{array}{l}\text { No. } \\
\text { tested }\end{array}$ & $\begin{array}{c}\text { No. } \\
\text { positive }\end{array}$ & $\begin{array}{c}\text { No. } \\
\text { doubtful }^{*}\end{array}$ & $\begin{array}{c}\text { Observed } \\
\text { prevalence } \\
(\%)\end{array}$ & $\begin{array}{c}\text { True } \\
\text { prevalence } \\
(\%)\end{array}$ & $\begin{array}{l}\text { 95\% } \\
\text { C.I. }{ }^{\dagger}\end{array}$ & $P$ value \\
\hline \multirow[t]{2}{*}{ Age } & $\leq 1$ year & 103 & 1 & 0 & 1.0 & 1.0 & $0.0-5.1$ & \multirow[t]{2}{*}{$<0.001$} \\
\hline & $\geq 2$ years & 455 & 18 & 2 & 4.0 & 4.1 & $2.5-6.4$ & \\
\hline \multirow[t]{3}{*}{ Sex } & Male & 59 & 0 & 0 & 0.0 & 0.0 & $0.0-6.2$ & \multirow[t]{3}{*}{0.001} \\
\hline & Female & 319 & 6 & 0 & 1.9 & 2.0 & $0.8-4.1$ & \\
\hline & Castrated & 180 & 13 & 2 & 7.2 & 7.4 & $4.0-12.4$ & \\
\hline \multirow{2}{*}{$\begin{array}{l}\text { Type of } \\
\text { cattle }\end{array}$} & Beef & 340 & 14 & 2 & 4.1 & 4.2 & $2.5-7.0$ & \multirow[t]{2}{*}{0.175} \\
\hline & Dairy & 218 & 5 & 0 & 2.3 & 2.4 & $0.9-5.3$ & \\
\hline \multirow[t]{4}{*}{ Region } & Northern & 188 & 1 & 0 & 0.5 & 0.5 & $0.0-2.8$ & \multirow[t]{4}{*}{0.009} \\
\hline & Central & 151 & 6 & 1 & 4.0 & 4.1 & $1.8-8.6$ & \\
\hline & Southern & 112 & 8 & 1 & 7.1 & 7.3 & $3.3-13.9$ & \\
\hline & Jeju Island & 107 & 4 & 0 & 3.7 & 3.8 & $1.3-9.3$ & \\
\hline Total & & 558 & 19 & 2 & 3.4 & 3.5 & $2.1-5.4$ & \\
\hline
\end{tabular}

${ }^{*}$ Doubtful results were regarded as negative for statistical analysis; ${ }^{\dagger} \mathrm{C}$. I.: Confidence interval

\section{Discussion}

Although a few studies (Lee et al., 1970; Lee, 1975) had been reported on B. besnoiti in Korean cattle with $3.8 \%$ and $6.7 \%$ prevalence rates which were slightly higher or similar to those found in the present study, a direct comparison among these data cannot be made as different diagnostic methods were used (ELISA vs. clinical signs). Evaluation of the seroprevalence of B. besnoiti ac- 
cording to age showed that adult cattle (age $\geq 2$ years) had a significantly higher seroprevalence compared to those aged $\leq 1$ year $(\mathrm{P}<0.001)$. The age dependence of B. besnoiti infection has been suggested in other studies (Lee et al., 1970; Lee, 1975; Álvarez-García et al., 2014; Gutiérrez-Expósito et al., 2014; Talafha et al., 2015). Talafha et al. (2015) stated that because B. besnoiti is transmitted via direct contact or by vectors, older individuals are more likely to get infected than younger ones. This may be a factor in the higher seroprevalence in cattle aged $\geq 2$ years.

Seroprevalence was also significantly greater $(\mathrm{P}=0.001)$ among castrated male cattle compared to females and non-castrated males. The association between sex and seropositivity has been controversial. Some authors found no association (Álvarez-García et al., 2014; Waap et al., 2014), others have reported higher seroprevalence in females (Ashmawy and Abu-Akkada, 2014), while others have reported greater susceptibility to besnoitiosis among males (Jacquiet et al., 2010). In this study, when castrated males and non-castrated males were counted together, seropositivity was greater in males than in females, and the difference was significant $(P=0.032)$. Further investigations will be necessary to clarify the association between sex and $B$. besnoiti infection.

In terms of the regional prevalence of $B$. besnoiti infection in Korea, with the exception of Jeju Island, there was higher seroprevalence among cattle in regions at lower latitudes. The lower latitudes of Korea have higher temperatures and total precipitation, which have been correlated to the abundance of flies, thereby suggesting that higher seropositivity among cattle in southern regions may be attributed to vector transmission. This tendency has also been observed in Europe, where the distribution of $B$. besnoiti is moving northward and spreading is due to the availability of vectors (Hornok et al., 2014). Jeju Island has a peculiar climate, and because of its geographical separation from other regions of the Korean peninsula, the seroprevalence in Jeju may have no relationship with the seroprevalence in other regions.

While beef cattle had higher seroprevalence than dairy cattle in the present study, the difference was not significant $(\mathrm{P}=0.175)$. Higher seroprevalence among beef cattle has been observed by some authors (Rinaldi et al., 2013; Álvarez-García et al., 2014), but not by others. Jacquiet et al. (2010) found that beef and dairy cattle were affected equally by besnoitiosis, while Álvarez-García et al. $(2013,2014)$ suggested that higher seroprevalence among beef cattle was related to the sampling region or husbandry conditions, but not to the breed.

This is the first study to report the seroprevalence of $B$. besnoiti in cattle in Korea using ELISA. The results suggest that $B$. besnoiti is widely distributed in Korea. Considering the intensive cattle husbandry systems being practiced and the regionally different seroprevalence of $B$. besnoiti in cattle in Korea, continuous monitoring and vector control are needed to reduce economic losses due to bovine besnoitiosis in the country. 


\section{References}

Álvarez-García, G., Fernández-García, A., Gutiérrez-Expósito, D., Ruíz-Santa Quiteria, J. A., Aguado-Martínez, A. and Ortega-Mora, L. M. (2014): Seroprevalence of Besnoitia besnoiti infection and associated risk factors in cattle from an endemic region in Europe. Vet. J. 200, 328-331.

Álvarez-García, G., Frey, C. F., Mora, L. M. O. and Schares, G. (2013): A century of bovine besnoitiosis: an unknown disease re-emerging in Europe. Trends Parasitol. 29, 407-415.

Ashmawy, K. I. and Abu-Akkada, S. S. (2014): Evidence for bovine besnoitiosis in Egypt -first serosurvey of Besnoitia besnoiti in cattle and water buffalo (Bubalus bubalis) in Egypt. Trop. Anim. Health Prod. 46, 519-522.

Cortes, H., Leitao, A., Gottstein, B. and Hemphill, A. (2014): A review on bovine besnoitiosis: a disease with economic impact in herd health management, caused by Besnoitia besnoiti (Franco and Borges, 1916). Parasitology 141, 1406-1417.

EFSA (2010): Bovine besnoitiosis: An emerging disease in Europe. EFSA J. 8, 1499.

García-Lunar, P., Ortega-Mora, L. M., Schares, G., Gollnick, N. S., Jacquiet, P., Grisez, C., Prevot, F., Frey, C. F., Gottstein, B. and Álvarez-García, G. (2013): An inter-laboratory comparative study of serological tools employed in the diagnosis of Besnoitia besnoiti infection in bovines. Transbound. Emerg. Dis. 60, 59-68.

Gutiérrez-Expósito, D., Esteban-Gil, A., Ortega-Mora, L. M., García-Lunar, P., Castillo, J. A., Marcén, J. M. and Alvarez-García, G. (2014): Prevalence of Besnoitia besnoiti infection in beef cattle from the Spanish Pyrenees. Vet. J. 200, 468-470.

Hornok, S., Fedák, A., Baska, F., Basso, W., Dencső, L., Tóth, G., Szeredi, L., Abonyi, T. and Dénes, B. (2015): Vector-borne transmission of Besnoitia besnoiti by blood-sucking and secretophagous flies: epidemiological and clinicopathological implications. Parasit. Vectors 8, 1-6.

Hornok, S., Fedák, A., Baska, F., Hofmann-Lehmann, R. and Basso, W. (2014): Bovine besnoitiosis emerging in central-eastern Europe, Hungary. Parasit. Vectors 7, 20.

Jacquiet, P., Liénard, E. and Franc, M. (2010): Bovine besnoitiosis: epidemiological and clinical aspects. Vet. Parasitol. 174, 30-36.

Lee, H. S. (1975): Studies on the infection of Besnoitia besnoiti among cattle in Korea [in Korean, with English abstract]. J. Inst. Dev. Livest. Prod. 3, 1-20.

Lee, H. S., Bak, U. B., Moon, M. H. and Shin, J. U. (1970): Studies on bovine besnoitiosis in Korea II. A survey on incidence in the enzootic region [in Korean, with English abstract]. Korean J. Parasitol. 8, 76-80.

Lee, S., Jung, B. and Kwak, D. (2015): Evidence of Anaplasma spp. exposure in native Korean goats (Capra hircus coreanae). Vet. Med. 5, 245-252.

Lee, S. H., VanBik, D., Kim, H. Y., Cho, A., Kim, J. W., Byun, J. W., Oem, J. K., Oh, S. I. and Kwak, D. (2016): Prevalence and molecular characterisation of Giardia duodenalis in calves with diarrhoea. Vet. Rec. 178, 633.

Oh, J., Lee, S. H., Lee, S. J., Kim, Y. H., Park, S. C., Rhee, M. H., Kwon, O. D., Kim, T. H. and Kwak, D. (2016): Detection of antibodies against Toxoplasma gondii in cattle raised in Gyeongbuk province, Korea. J. Food Prot. 79, 821-824.

Olias, P., Schade, B. and Mehlhorn, H. (2011): Molecular pathology, taxonomy and epidemiology of Besnoitia species (Protozoa: Sarcocystidae). Infect. Genet. Evol. 11, 1564-1576.

Reiczigel, J., Földi, J. and Ózsvári, L. (2010): Exact confidence limits for prevalence of a disease with an imperfect diagnostic test. Epidemiol. Infect. 138, 1674-1678.

Rinaldi, L., Maurelli, M. P., Musella, V., Bosco, A., Cortes, H. and Cringoli, G. (2013): First crosssectional serological survey on Besnoitia besnoiti in cattle in Italy. Parasitol. Res. 112, $1805-1807$. 
Schares, G., Langenmayer, M., Scharr, J., Minke, L., Maksimov, P., Maksimov, A., Schares, S., Bärwald, A., Basso, W. and Dubey, J. (2013): Novel tools for the diagnosis and differentiation of acute and chronic bovine besnoitiosis. Int. J. Parasitol. 43, 143-154.

Talafha, A. Q., Al-Majali, A. M., Ababneh, M. M. and Abutarbush, S. M. (2015): Epidemiologic study on Besnoitia besnoiti infection in dairy herds in Jordan. Parasitol. Res. 114, 24912497.

Thrusfield, M. (2005): Surveys. In: Thrusfield, M. (ed.) Veterinary Epidemiology. Blackwell Publishing, Oxford, UK. pp. 228-246.

Waap, H., Nunes, T., Cortes, H., Leitão, A. and Vaz, Y. (2014): Prevalence and geographic distribution of Besnoitia besnoiti infection in cattle herds in Portugal. Parasitol. Res. 113, 37033711 . 